\title{
EXPERIENCIA DE INNOVACIÓN EN DESARROLLO PROFESIONAL DOCENTE*
}

\author{
INNOVATION EXPERIENCE IN TEACHER PROFESSIONAL DEVELOPMENT
}

\author{
Jorge Rodríguez-Sosa**, Susana K. Lingan ${ }^{* * *}$, Ronald M. HeRnández ${ }^{* * *}$ Y \\ J'JOEL ALHUAY-QUISPE ${ }^{* * * * *}$
}

\begin{abstract}
*Este estudio forma parte del Programa de Especialización en Tutoría y Acompañamiento Pedagógico y fue realizado en el marco del Programa Nacional de Formación y Capacitación Pedagógica (PRONAFCAP). ${ }^{*}$ Magister en Sociología. Director del Centro de Investigación de la Universidad San Ignacio de Loyola (USIL).

Lima, Perú. E-Mail: jrodriguezs@usil.edu.pe - ORCID: http://orcid.org/0000-0002-8440-4891

***Licenciada en Psicología. Docente y Coordinadora Académica de la Carrera de Psicología en la Universidad San Ignacio de Loyola (USIL). Lima, Perú. E-Mail: klingan@usil.edu.pe

****Licenciado en Psicología. Coordinador de Publicaciones Científicas del Centro de Investigación de la Universidad San Ignacio de Loyola (USIL). Lima, Perú. E-Mail: rhernandezv@usil.edu.pe

${ }^{* * * * * B}$ achiller en Bibliotecología y Ciencias de la Información. Asistente de Investigación del Centro de Investigación de la Universidad San Ignacio de Loyola (USIL). E-Mail: jalhuay@usil.edu.pe ORCID: http://orcid.org/0000-0002-1903-4687

Facultad de Educación de la Universidad Peruana Cayetano Heredia. Lima - Perú.
\end{abstract}

\section{RESUMEN}

Las escuelas latinoamericanas enfrentan demandas desde los entornos en los que operan, que definen escenarios caracterizados por las brechas entre aquello que las escuelas están capacitadas para ofrecer (representado principalmente por los docentes en el aula) y las necesidades emergentes de estudiantes que responden a entornos sociales concretos y muy diversos. Cerrar esta brecha es una prioridad y se estima que para ello es necesario fortalecer las capacidades de los docentes en aspectos como la contextualización de sus prácticas. Para ello se ejecutaron diversas intervenciones empleando distintos formatos de capacitación y metodologías de trabajo. Se presenta un estudio observacional realizado sobre una experiencia de desarrollo profesional docente que incorporó un componente de investigación-acción, con 21 docentes en servicio. El propósito del estudio fue establecer cómo y cuánto se fortalecieron las habi- lidades y expectativas que componen la competencia de investigación docente en el grupo mencionado. Se emplearon distintas herramientas para juntar información como pruebas, grupos focales, análisis de testimonios y de textos. Asimismo, se utilizaron complementariamente análisis cuantitativo y cualitativo, con énfasis en este último.

Los resultados dan cuenta de mejoras significativas en las habilidades de problematización, empleo de evidencias y herramientas metodológicas, formulación de hipótesis y estrategias de intervención y redacción científica; así como de mejoras igualmente significativas en la competencia de investigación en general. Los mismos no reportan cambios o mejoras en las expectativas de empleo de la investigación en la enseñanza.

Palabras clave: Investigación-acción; Competencia de investigación; Habilidades de investigación; Expectativas; Formación docente; Docentes en servicio. 


\section{ABSTRACT}

Currently many schools in Latin America face strong demands from their environments, these demands are marked by an increasing and more complex economic poverty, a deeper social inclusion and a lack of social cohesion, which are reflected in an escalated individualism. In addition to these demands, there are other expectations imposed by the academic and political world. In the academic world, contextualized learning and quality education services are expected, while in the political world, the demands are constituted by more levels of inclusion and of student permanence in schools. Together, these demands define a scenario for education characterized by several gaps: the ones between the performances of students from public and private schools, urban and rural schools, considering if they are male or female students, amongst others. It is important to highlight one of the most substantial disparity, which is the breach that exists from what the school is able to offer, greatly represented by the teacher in the classroom, and the emerging needs of the students, these needs correspond to particular and very diverse social environments, where every specific demand must be satisfied. To Close this gap, and the others previously mentioned, which are of no less importance, a priority in the political agenda of the governments must be given. To accomplish this major point, it is necessary to strengthen teachers' abilities to use and manage technologies and tools that will allow them to cater to the diversity of students in each of the schools attended, as well as contextualize teaching practices, and adapt them to reach the needs of these specific groups. In order to address these points many interventions, which used various training modes and work methodologies, were executed. However the actions taken, they have not have the desired outcome until now. Moreover the evidence resulting from international research specify that to strengthen the skills of the teachers accordingly, it is necessary that the training of in-service teachers should allow the promotion of spaces for reflection and dialog, as well as the opportunity to question common knowledge and practices. Data obtained from the field report that training given to teachers only generates real changes in teaching practices when these teachers have previously changed their understanding and beliefs of what teaching is to them, aligning them with the new approaches. This same data also reports that one of the most effective methodologies to generate these positive changes is the use of action research.

The following observational investigation composed a study group of 21 in-service teachers attending a professional development that incorporated an action-research component. The purpose of this study is to establish how, and to what extent, the capacities for competitive research were strengthened in the group previously mentioned. To achieve this, different information collection tools were used for the observation of teaching performance: Tools such as tests, focus groups, analysis of testimonies and products. Complementary methodologies were also used for the quantitative and qualitative analysis, giving an emphasis on the latter. The results obtained from the quantitative analysis account for a significant improvement in each one of the seven skills that were part of this research, which are presented in the study as a whole. The results of the qualitative analysis report improvements in areas such as the formulation of problems, the use of evidences and methodological tools, hypothesis formulation and intervention, and scientific writing strategies, as well as important improvement in the capacity to research generally speaking. However, the outcome of this investigation has not created and impact in teachers so that will pursue them to implement and evaluate the use of research in their teaching practices.

Key words: Action research; Research competence; Research skills; Expectations; Teacher training; In-service teachers.

\section{INTRODUCCIÓN}

Actualmente las escuelas latinoamericanas enfrentan escenarios de alta complejidad, incluso pueden parecer hostiles. La realidad cotidiana les exige responder a entornos sociales donde la pobreza es cada vez mayor y 
más compleja, la exclusión social es más profunda y la falta de cohesión se manifiesta en un creciente individualismo (López, 2009). La política les exige logros en accesos, aprendizajes y permanencias (Fernandes, 2014), por lo menos equivalentes a los alcanzados en otras partes del mundo; mientras la academia otro tanto, en relevancia y contextualización de los aprendizajes (Herrington, Yezierski, Luxford, K.M. \& Luxford, C.J., 2011) y calidad de los servicios (Aranguren, 2007). Esas exigencias definen escenarios educativos diferentes, que emergen de las nuevas circunstancias y que progresivamente se alejan de aquellos a los que las escuelas tenían que dar respuesta, en la medida que fueron concebidas desde y para ellos. Así, en las escuelas se camina a tientas intentando sortear las brechas entre lo que se está en capacidad de ofrecer (representado en gran parte por el docente en el aula) y las necesidades emergentes de los estudiantes, situadas en el entorno social concreto en el que se las debe satisfacer.

Diversos estudios en América Latina reportan realidades en las cuales los diseños curriculares están fragmentados y mantienen escasa vinculación con los contextos escolares en los que el docente lleva a cabo su práctica (Rodríguez, 2004; Vaillant, 2009). Tal situación le exige al docente una diversidad de capacidades y herramientas de atención con las que no cuenta. Por ello, es lógico deducir que la crisis de las escuelas se explica en buena cuenta por lo limitado de la capacidad de sus docentes para dimensionar la magnitud de los cambios (López, 2009), capacidades que también serían necesarias para estructurar respuestas que aseguren un mínimo de ajuste entre las ofertas institucionales y las necesidades locales.

El perfil necesario del docente es uno orientado hacia el logro de una práctica reflexiva y contextual (Chacón, M.A., Chacón, T. \& Alcedo, 2012), tomando en cuenta que la enseñanza se ha tornado una actividad poco predecible, que es parte de un proceso incierto y marcado por la complejidad (Perrenoud, 2004), fenómeno que también se manifiesta a nivel de la docencia universitaria
(Laudadío, 2012). Optimizar las herramientas de aprendizaje conduce a una mejor enseñanza, si se asume una postura reflexiva y crítica sobre lo que se hace. En ese marco, ¿qué podría facilitar o impedir el logro de este perfil docente? ¿En qué condiciones se pueden dar los cambios? ¿Es posible alcanzarlos con los programas de formación en servicio actuales, tal y como están diseñados, o se requieren disposiciones organizacionales especiales para ello?

Como es sabido, los docentes tienen ideas y creencias sobre cómo debe ser la enseñanza que son difíciles de cambiar. La investigación previa reporta sobre el importante papel que juegan los conocimientos y las creencias que traen los docentes en la enseñanza que brindan. También sobre el hecho de que capacitarlos en nuevas metodologías no asegura que luego plasmen lo aprendido en cambios en sus prácticas (Herrington et al., 2011). Para que ello ocurra, esas capacitaciones deben incluir espacios para el cuestionamiento de conocimientos y creencias, entendiendo que la adopción de cualquier nueva metodología requiere una base de conocimientos y de creencias alineadas con esas orientaciones, como una condición que pueda gatillar la disposición de los docentes a cambiar sus prácticas. Lamentablemente, la formación de docentes en servicio se limita fundamentalmente al fortalecimiento de contenidos disciplinares y / o de metodologías didácticas, operando desde enfoques remediales y lógicas prescriptivas (Gonzalez-Weil, Cortez, Pérez, Bravo \& Ibaceta, 2013), sin incorporar (o haciéndolo muy tangencialmente) los aprendizajes de las experiencias previas más exitosas, que tienen que ver con la disposición de espacios para el trabajo colaborativo, que favorezcan la reflexión, el intercambio de buenas prácticas y el cuestionamiento de los propios conocimientos y creencias (Park Rogers et al., 2007).

Estos aprendizajes muestran que dar a los docentes la oportunidad de cuestionar sus propias prácticas y las creencias que las sostienen, facilita que asuman posturas reflexivas y abiertas, condición necesaria para producir los cambios deseados (Chamizo \& García-Franco, 2013). 


\section{LA COMPETENCIA DE INVESTIGACIÓN DOCENTE}

Distintos autores (García Fraile \& Tobón Tobón, 2008; Irigoin \& Vargas, 2002; Tobón, 2005 ) definen a las competencias como procesos complejos que las personas ponen en acción-actuación-creación para resolver problemas y realizar actividades de distinta índole, aportando con ello a la construcción y transformación de la realidad. En esos procesos se integran el saber ser (motivación, iniciativa, expectativas, trabajo colaborativo), el saber conocer (observar, cuestionar, analizar, explicar) y el saber hacer (desempeño basado en procedimientos y habilidades), teniendo en cuenta los requerimientos del entorno, las necesidades personales (e intereses) y la incertidumbre. Es competente quien dispone de los conocimientos, habilidades y actitudes necesarias para resolver con autonomía y flexibilidad los problemas propios de su campo, sea en una profesión, oficio u otro trabajo. En el campo de la investigación de la docencia, se puede decir que la competencia investigativa resulta de integrar adecuadamente los conocimientos (teorías y principios en el campo pedagógico y disciplinar), las habilidades (procedimientos metodológicos de uso recurrente en investigación) y las actitudes (reflexión, perspectiva crítica, apertura al cambio), que son necesarios para el empleo del método científico en el análisis, la comprensión y la transformación de realidades que son parte de las prácticas docentes y del currículo.

\section{LA INVESTIGACIÓN-ACCIÓN}

Algunas de las características que se le atribuyen a la investigación-acción son las siguientes: emplea sistemáticamente la autoinspección de la práctica y lo hace desde una perspectiva crítica (Carr \& Kemmis, 1988), toma a la práctica docente como objeto de estudio y campo de aprendizajes (Mitchener \& Jackson, 2012), aborda problemas cuya solución supone optimizar los aprendizajes (Montecinos, Solis \& Gabriele, 2001) y pone énfasis en el protagonismo del docente en los procesos de investigación (McKernan,
1996). Como vemos, la investigación-acción es una metodología fuertemente asociada a la mejora continua de las prácticas docentes.

En ella se produce una relación dialéctica entre la reflexión (generación de conocimiento, teoría) y la acción (práctica, cambio), que supera la relación lineal y asimétrica que va de la reflexión a la acción, muy común en la investigación académica convencional. En esta metodología, la reflexión y la acción se dan en momentos simultáneos, interactúan y se retroalimentan en un proceso circular permanente. Ambas cumplen funciones necesarias, ambas son indispensables, pero siempre la acción juega el rol central, prioridad que es consistente con lo sostenido por Díaz, Villalón y Adlerstein (2015), para quienes, en el campo de la educación, el conocimiento práctico del docente termina definiendo el conocimiento en general que ellos producen.

\section{LA NUEVA FORMACIÓN DOCENTE}

Ibernón (2001) sostiene que la formación de docentes más adecuada es aquella que se da en colectivos que emplean sistemáticamente la reflexión deliberativa en el marco metodológico de la investigación-acción. Más aún si la finalidad de ello es promover la búsqueda de nuevas comprensiones sobre la práctica docente en la práctica misma (Montecinos et al., 2001). Una revisión documental hecha por Zeichner (2001) presenta casos en que la investigación-acción, en ciertas condiciones, produce mejoras en las prácticas docentes. También es posible mostrar evidencia abundante de que el empleo de la investigación-acción, como un componente dentro de programas de formación docente de mayor alcance, se asocia al cambio de diversos aspectos de la docencia, como por ejemplo:

- el pensamiento reflexivo (Chacón et al., 2012; Maarof, 2007),

- la actitud indagatoria sobre la propia práctica (Justi \& Van Driel, 2005),

- el cuestionamiento de conocimientos y creencias en torno a la enseñanza o el aprendizaje (Herrington et al., 2011; Megovan-Romanowicz, 2010), 
- las habilidades de exploración e identificación de problemas en el aula (Yamin-Ali, 2010),

- el empleo de estrategias de enseñanza basadas en la práctica de la ciencia e investigación (Blanchard, Southerland \& Granger, 2009; Silverstein, Dubner, Miller, Glied \& Loike, 2009),

- la interacción entre docentes en la escuela, desde una perspectiva dialógica y reflexiva (Graebner, de Souza \& Saito, 2009),

- la contextualización de las actividades de aprendizaje (Ruiz-Mallen, Barraza, Bodenhorn, Ceja-Adame \& Reyes-García, 2010) y - las expectativas de empleo de la investigación en el aula (Blanchard, et al., 2009).

Sin embargo, esos resultados son indirectos desde la perspectiva de lo que se pretendía en este estudio, porque las intervenciones a las que hacen referencia tuvieron como propósito mejorar las destrezas pedagógicas específicas (en la enseñanza de la matemática o las ciencias, o en la adaptación de materiales, o en la organización del aula), sin proponerse (al menos no de manera explícita) el fortalecimiento de la dimensión investigativa. No se tiene evidencia sobre cómo y cuánto se fortalece la competencia de investigación de los docentes si se los expone a una intervención diseñada con ese fin que utilice metodologías dialógicas y participativas. Por ello, el objetivo de este estudio fue cubrir ese vacío ante el planteo y dar respuesta a la siguiente pregunta: ¿Cómo y cuánto cambian las habilidades de investigación y las expectativas sobre su empleo en la mejora de la docencia, en un grupo de docentes en servicio que participaron de una experiencia de innovación pedagógica que incorporó metodologías de investigación-acción?

\section{MÉTOdO}

Se realizó un estudio observacional de carácter analítico, acotado a la evaluación de los cambios en los conocimientos, el manejo de procedimientos metodológicos y las expectativas en torno a la investigación, experimentados por un grupo de docentes en servicio en el marco de su participación en el Programa de Especialización en Tutoría y Acompañamiento Pedagógico, programa que incorporó un componente de investigación-acción ${ }^{1}$.

El estudio se limitó a observar lo que ocurrió en el proceso de capacitación, no incluyendo la evaluación de las prácticas docentes en aula. El análisis de la información recolectada empleó métodos cuantitativos y cualitativos.

\section{Participantes}

Participaron 21 docentes (14 mujeres y 7 varones), de Lima y del norte de Perú (Barranca, Huacho, Casma, Chimbote, La Libertad). Las edades de 12 docentes se ubicaban en el rango de 30 a 45 años, siete tenían entre 46 y 55 años, y solo dos contaban con menos de 30 años. La mayoría (16) se formó en Institutos de Educación Superior Pedagógica y en cuanto a los grados y títulos obtenidos, cinco contaban con el grado de maestría, nueve con una segunda especialización y siete con el título profesional de educación.

TÉCNICAS E INSTRUMENTOS DE RECOLECCIÓN DE INFORMACIÓN

Se utilizaron diferentes técnicas que variaron según las fuentes (espacios, productos, informantes) en las que fueron aplicadas. Se empleó el análisis de testimonios sobre las grabaciones hechas en los círculos de reflexión y sesiones de taller como así también el análisis de textos en la evaluación de los productos. Finalmente, se trabajó con grupos focales y se aplicaron pruebas a los docentes, en momentos de entrada y salida, para estable-

1 Este programa, a su vez, los acreditaba como docentes formadores del Programa Nacional de Formación y Capacitación Pedagógica (PRONAFCAP), programa público de capacitación en servicio del Ministerio de Educación del Perú (MINEDU). 
cer cambios en los conocimientos, habilidades y expectativas.

Se diseñó una Prueba de Conocimientos y Habilidades de Investigación ad hoc para el estudio, que se aplicó individualmente en momentos antes y después para evaluar el fortalecimiento de la competencia de investigación de los docentes, empleando los procedimientos recurrentes en la metodología de investigación-acción. Se trató de una prueba abierta (no estructurada) que planteó una situación real en la educación peruana: la pobreza en los resultados de nuestras evaluaciones de desempeño de los estudiantes y las diversas brechas que estos resultados muestran. Luego, se propuso a los docentes que desde esa situación identificasen un problema que pudiera ser abordado desde la práctica en aula, así como sus posibles soluciones. Para la calificación se empleó una rúbrica, diseñada y revisada por expertos, cuyas puntuaciones estaban en un rango de 0 a 100. El énfasis se puso en criterios como la relevancia y la pertinencia del problema abordado, la forma de justificarlo y la viabilidad de las soluciones propuestas.

También se emplearon como fuentes de evidencia los textos producidos por los docentes. Con ellos se buscaba observar los cambios en las habilidades de problematización, el empleo de fuentes y evidencias y la redacción. Estos textos fueron entendidos como ejercicios de reflexión en los que los docentes exponían su comprensión de los problemas y las maneras de abordarlos. Adicionalmente, esos testimonios dieron cuenta de sus percepciones sobre distintos temas, así como de los cambios que desde sus perspectivas, experimentaron por efecto de su participación en el componente.

\section{Procedimientos \\ CONTEXTO DE LA OBSERVACIÓN: El COMPONENTE DE INVESTIGACIÓN-ACCIÓN}

El componente de investigación-acción fue incluido como parte de los servicios de una universidad privada de Lima en su rol de ente ejecutor del PRONAFCAP. No era parte del diseño original de este programa, más bien se trató de un valor añadido en la oferta formativa de la universidad.

Sus propósitos fueron: (1) que los docentes formadores entendiesen la importancia de incorporar el trabajo colaborativo, la reflexión crítica y la auto-inspección de la práctica, para luego poder sensibilizar a docentes de aula sobre la necesidad de ello y (2) que aprendieran la metodología de investigaciónacción seleccionada como una de las metodologías del trabajo en aula por el MINEDU.

La ejecución comprendió un total de 10 semanas, con una organización basada en tres módulos que sumaron 120 horas, 48 de estudios presenciales ( 32 de sesiones de taller y 16 de círculos de reflexión) y 72 de estudio independiente. El módulo Problematización empleó 24 horas presenciales y 40 de estudio independiente, mientras Búsqueda de información y evidencias y Análisis de causas, 12 horas presenciales y 16 de estudio independiente cada uno. El esquema de todos los módulos fue el mismo: talleres y círculos de reflexión, ambos presenciales, además del estudio independiente antes, durante y después de la etapa presencial, que básicamente fue empleado en la elaboración de los productos de cada módulo. El Cuadro 1 muestra la relación de las dimensiones de la investigación-acción trabajadas en el componente y las habilidades fortalecidas.

Entre las habilidades desarrolladas, cinco de ellas corresponden a procedimientos comunes en la metodología de investigaciónacción: (1) la identificación de problemas, (2) el análisis de causalidad, (3) el empleo de fuentes, informantes y técnicas, (4) la formulación de hipótesis de acción y (5) la propuesta de estrategias de intervención (Rodríguez Sosa, 2005). Las otras dos son habilidades transversales a la investigación en general: la búsqueda de información y evidencias y la redacción científica. También se trabajó en las expectativas de los docentes sobre el empleo de la investigación en la mejora de las prácticas. En todo momento se trató de alinear la definición de investigación-acción empleada, lo ofrecido en el componente y lo que se terminó evaluando. 


\section{PROCEDIMIENTOS PARA LA OBSERVACIÓN Y EL ANÁLISIS}

A nivel cuantitativo, la competencia de investigación fue evaluada como una totalidad y por cada una de las siete habilidades involucradas, a través de la Prueba de Conocimientos y Habilidades de Investigación. Luego se extrajeron los estadísticos descriptivos sobre los puntajes totales, mientras que a nivel bivariado, se empleó la prueba $t$ de grupos relacionados, que requirió la aplicación previa de pruebas de normalidad para muestras pequeñas como Shapiro-Wilk. Se procesaron los datos recogidos con el programa IBM SPSS Statistics versión 18 (IBM, CA, USA).

A nivel cualitativo, se trabajó con las siete habilidades y las expectativas, empleando testimonios y textos. Para el tratamiento de los testimonios se emplearon matrices de doble entrada: en la entrada vertical los docentes y en la horizontal, los temas abordados. Sobre esa información, en la que ya se había separado aquella data que no era de utilidad, se aplicaron los procedimientos convencionales del análisis cualitativo: reducción de datos, presentación de datos e interpretación (Miles \& Huberman, 1984). Para el tratamiento de los textos que eran parte de los productos intermedios y del producto final, se utilizaron criterios como por ejemplo: qué tan pertinente es el problema abordado, qué tan relevante es y qué tan fundamentado se presenta. Según este enfoque, un problema es pertinente cuando es identificable en las prácticas docentes cotidianas y puede ser abordado desde la práctica misma (Rodríguez Sosa, 2005). Es relevante cuando se justifica por la potencial introducción de beneficios (y de beneficiarios) y / o por sus implicancias prácticas en la solución de problemas (Hernández, Fernández-Collado \& Baptista, 2007). Y está fundamentado cuando se basa en evidencias recogidas de la investigación previa en el campo (Slafer, 2009).

En ambos casos, testimonios y textos, se puso especial énfasis en la observación de los cambios (aquello que fue mejorado, sea por la intervención o por algún proceso emergente en el marco de la experiencia) y las per- manencias (aquello que no se pudo cambiar, al margen de la intencionalidad de la intervención), en el manejo de conceptos y de procedimientos y en las expectativas.

\section{Resultados}

El análisis cuantitativo comprendió la evaluación de la competencia de investigación como totalidad, y de cada una de las siete habilidades que la componen, según la definición utilizada en este estudio.

El análisis cualitativo comprendió la descripción de los cambios en esas mismas habilidades y en las expectativas de empleo de la investigación en la docencia.

Con la finalidad de facilitar la comunicación de estos resultados, se presentan agrupados en: (1) competencia de investigación (análisis cuantitativo), (2) identificación de problemas y análisis de causalidad, (3) empleo de evidencias y herramientas de información, (4) hipótesis de acción y estrategias de intervención, (5) redacción y (6) expectativas de empleo de la investigación (análisis cualitativo).

\section{1.- LA COMPETENCIA DE INVESTIGACIÓN}

La evaluación de la competencia de investigación empleó la información obtenida con la Prueba de Conocimientos y Habilidades de Investigación que se aplicó en momentos antes y después de la intervención. El uso del estadístico Shapiro Wilk evidenció que la distribución de puntuaciones se acercaba a la distribución normal, por lo que se empleó el estadístico $t$ de grupos relacionados en la comparación de momentos. La diferencia de las puntuaciones medias entre los momentos antes (32.24 puntos) y después (64.81 puntos) fue mayor a $32(-32.57)$, diferencia estadísticamente significativa $[t(20)=-24.51$, $p<.0001]$.

La evaluación también arrojó que para cada habilidad los puntajes fueron mayores en el momento después, con diferencias que resultaron estadísticamente significativas en todos los casos (ver Tabla 1). 
2.- LA IDENTIFICACIÓN DE PROBLEMAS Y EL ANÁLISIS DE CAUSALIDAD

Una de las primeras actividades solicitadas a los docentes que participaron del componente de investigación-acción, fue que identificaran un problema en su práctica profesional que representara una limitación. Frente a ello, un hecho que llamó la atención del equipo a cargo de la intervención fue la tendencia de los docentes a buscar las causas de los problemas en ámbitos externos a la práctica docente.

Algunas causas recurrentes fueron los rasgos de personalidad de los estudiantes: 'jóvenes que tienen muy poca motivación por el estudio, o no tienen ninguna' (Sesión de Taller $\mathrm{N}^{\circ} 1$ ). También lo limitado de sus capacidades: 'Es difícil que puedan tener algún aprendizaje si comprenden la mitad de lo que leen o menos, entonces cómo esperamos que entiendan lo que se les pide resolver en un problema' (Sesión de Taller $N^{\circ} 1$ ). O problemas en las familias: 'A ese muchacho lo condenaba el que su familia fuera muy pobre. Él puede tener capacidad para el estudio pero eso no es lo importante en su familia [...] la prioridad, es que trabaje en cualquier cosa y traiga algún ingreso adicional a la casa, no la escuela' (Sesión de Taller $N^{\circ} 1$ ). También se refirieron frecuentemente a otras causas ubicadas fuera de la escuela e incluso externas al mundo de la educación, tales como la pobreza, la desnutrición, la violencia social o la crisis de valores en las familias.

Los problemas identificados tuvieron con algunas variantes, un sentido similar al siguiente: '¿Por qué en los espacios no convencionales de trabajo en aula (colectivos, participativos, lúdicos) se genera desorden y se exacerba el bullying en estudiantes del VI ciclo de educación secundaria de la Institución Educativa María Negrón Ugarte de Trujillo?' (Primer producto intermedio). Las causas expuestas para este problema fueron: 'los entornos sociales violentos; la violencia cotidiana que ha entrado en la escuela; los altos niveles de desestructuración en las familias que no favorecen la transmisión de reglas de convivencia ni el respeto a normas básicas; o la crisis de los valores sociales que antes eran importantes', todas ellas con similares niveles de incidencia. Como se puede ver, estas causas se ubican en un otro externo a la escuela que supuestamente tiene capacidad de influir en lo que se hace en ella. Esta tendencia lleva a que se esquive una necesaria reflexión sobre la práctica profesional, ejercicio que podría conducir a los docentes a cuestionarse sobre aquello que se hace y a buscar las causas de los problemas en deficiencias que se pueden hallar en la docencia misma.

Los círculos de reflexión jugaron un rol fundamental en ese propósito. Desde la programación se tuvo previsto que el componente incluyese cuatro momentos de cuatro horas cada uno para estos espacios. Sin embargo, la necesidad llevó a que, conforme avanzaba la ejecución, buena parte de las horas de taller se tornasen a ese formato reflexivo, superando largamente las 16 horas originalmente previstas y dándole un carácter reflexivo, crítico y de negociación a gran parte de los procesos de capacitación.

En el primer círculo de reflexión se planteó como situación para el análisis ¿Qué hacer ante las diferencias (en desempeños, motivaciones, intereses u otras) al interior de las aulas? Este ejercicio fue una excelente plataforma para la reflexión y el cuestionamiento, en el marco de un diálogo abierto. Conforme avanzaba la ejecución, se pasó progresivamente del 'tenemos este problema, con estas causas y con estas características, que limita lo que podemos hacer como docentes', a un plano distinto que se cuestionaba sobre 'qué cambios en las maneras como hacemos las cosas (porque no hemos tenido la disposición o las capacidades necesarias) supone el abordar efectivamente ese tipo de problemas'. Este nuevo acercamiento incorporó intencionalidad pedagógica a las soluciones, haciendo que el docente juegue un rol protagónico en ellas y que su práctica sea el espacio de operaciones. Así el problema planteado por el Docente 18 en el primer producto intermedio, presentado párrafos arriba, fue modificado y quedó planteado como sigue: ¿Cómo consensuar y explicitar actividades, normas y acuerdos para aprovechar al máximo los espacios no convencionales de trabajo en aula (colectivos, 
participativos, lúdicos) con los estudiantes del VI ciclo de educación secundaria de la Institución Educativa María Negrón Ugarte de Trujillo? (Segundo producto intermedio: Docente 18). Con causas que tenían que ver fundamentalmente con 'carencias en el manejo de metodologías para el trabajo en grupos, el poco conocimiento de dinámicas que favorezcan la participación de los estudiantes', o con 'no se cuenta con herramientas suficientes para despertar interés y motivación'.

Los nuevos abordajes sugieren que los docentes fueron encontrando los factores explicativos y las posibilidades de solución de los problemas en zonas cada vez más cercanas a sus ámbitos de influencia, en sus prácticas. También sugieren que en lo actitudinal asumieron posturas más favorables a la autoinspección de las prácticas, transitando desde problemas percibidos como carencias en los estudiantes, sus familias o el entorno, a problemas percibidos como deficiencias o necesidades a ser cubiertas que se podían hallar en aquello que el docente hace.

\section{3.- EL EMPLEO DE EVIDENCIAS Y HERRAMIENTAS DE INFORMACIÓN}

Al inicio de la intervención varias de las propuestas de problema presentadas fueron justificadas apelando a experiencias personales como toda evidencia, sin complementarlas con evidencia real proveniente de la investigación o la evaluación sistemática. En otros casos, se empleó información cuestionable, no referenciada, o se intentó sustentar los problemas con argumentos particulares, muy parciales, generalmente apoyados en las creencias que los docentes traían o en las teorías implícitas que manejan.

La mayoría de las propuestas empleó una o dos fuentes secundarias indirectas (documentos institucionales, informes sectoriales o textos de divulgación), acompañadas de un tratamiento de datos muy pobre. En algunos casos, cuando los datos no se alineaban con las teorías preestablecidas, se los tomó mutilados, distorsionados o simplemente, se los dejó de lado. No hubo propuestas en las que fuera evidente que el autor tuviera conciencia de que los problemas abordados involucraban a distintos actores (estudiantes y colegas como mínimo, pero también padres y autoridades), con distintas perspectivas sobre los hechos y distintos intereses. En ninguna propuesta a nivel del primer producto intermedio se utilizaron procedimientos de triangulación de fuentes, sumando a la información secundaria disponible el empleo de entrevistas, grupos focales $\mathrm{u}$ otras técnicas de interacción con los informantes.

Esta situación dio cuenta de la necesidad de acercar a los docentes al empleo de otras fuentes de información y al análisis de diversas perspectivas. Para ello, fue necesario discutir el tema con ellos, acordando el fortalecimiento de sus habilidades de búsqueda y recopilación de información, así como de análisis. Esto se hizo principalmente en las sesiones 5 y 6 de taller del módulo Búsqueda de Información y Evidencias, donde se promovieron presentaciones orales para que cada docente expusiese su propuesta, deteniéndose en la evaluación de la evidencia que la sustentaba, lo que hizo posible discutir la necesidad de incluir el discurso de otros actores escolares en la justificación de las propuestas. En el segundo y tercer producto intermedio se empezaron a incorporar otras perspectivas, pero fue en el producto final donde 12 de los 21 docentes incorporaron claramente perspectivas múltiples y triangulación de fuentes, mientras siete docentes adicionales también lo hicieron aunque con menor claridad.

\section{4.- LAS HIPÓTESIS DE ACCIÓN Y ESTRATEGIAS DE IN- TERVENCIÓN}

En la formulación de hipótesis de acción se mantuvo el error observado a nivel de la problematización. Los docentes tendían a ubicar las causas de los problemas en ámbitos externos a sus prácticas, por ende las soluciones propuestas también debían procesarse fuera de la escuela, bajo la responsabilidad de actores distintos a los actores escolares. Este abordaje de los problemas sesgaba el conocimiento de las situaciones, limitando de paso la posibilidad de anticipar estrategias de solución efectivas, pues se operaba frente a pro- 
blemas que no existian en la escuela o que se percibían muy distorsionados.

La propuesta de problema del Docente 18 ya presentada, cambió radicalmente entre el primer y segundo producto intermedio, pasando de un enfoque donde el rol del docente no era activo: 'los espacios no convencionales de trabajo en aula (colectivos, participativos, lúdicos) generan desorden y exacerban el bullying entre los estudiantes', a otro en que la solución pasa por la actividad y el protagonismo del docente: 'qué se puede hacer (qué cambios se pueden operar) para aprovechar los espacios no convencionales de trabajo en aula'. Este último abordaje ubicó el problema y sus causas en carencias que el docente tenía en el manejo de herramientas para el trabajo en grupos, la participación de los estudiantes o la motivación. Así las estrategias de intervención se enfocaron en el fortalecimiento de esas capacidades: la implementación de un taller en didácticas participativas con docentes; el rediseño organizacional del aula, previendo un espacio de diálogo, acuerdos y toma de decisiones; darle capacidades de decisión a los estudiantes para seleccionar los temas clave que serán abordados desde los espacios no convencionales (Producto final: Docente 18).

A lo largo del componente otras propuestas de los docentes transitaron esa misma ruta, con velocidades y claridad variables, pero con el mismo sentido del cambio. De los 19 docentes que presentaron el producto final (proyectos de mejoramiento educativo), 14 diseñaron una estrategia de intervención realista, sustentada y viable.

\section{5.- LA REDACCIÓN}

En el campo de la redacción hubo algunos resultados sorprendentes. Si bien es sabido que la lectura comprensiva y la producción de textos no son precisamente fortalezas profesionales de los docentes latinoamericanos, no se tenía mayor información acerca de que la redacción científica o la comunicación de resultados de investigación fuese un tema desconocido para ellos. En el primer producto in- termedio e incluso en el segundo se pudo observar que los docentes tenían una redacción muy deficiente, además de un empleo escaso o nulo de referencias para dar sustento a lo que se argumentaba. Sumado a ello, no se utilizaba estándar de redacción alguno, ni para el sistema de citas, la colocación de referencias, o la inclusión de tablas, figuras u otros. Algunos de los textos presentados, al margen de la relevancia o no del problema abordado, tendían a una descripción muy detallada, casi literaria, de situaciones que poco tenían que ver con el tema que se estaba abordando, o a un estilo panfletario, o se asemejaban a telegramas o conjuntos de guiones con poca conexión entre sí y menos con el tema general.

Sin embargo, en este campo se alcanzaron mejoras importantes y en plazos relativamente cortos. En el producto final, 9 de los 19 docentes que entregaron el manuscrito utilizaba el lenguaje claro, directo y sintético que debe ser empleado en la comunicación científica, por supuesto con niveles variables de calidad. Asimismo, 13 docentes referenciaban sus argumentos y emplearon evidencias, mientras 16 utilizaban adecuadamente citas, referencias y auxiliares.

\section{6.- LAS EXPECTATIVAS DE EMPLEO DE LA INVESTI- GACIÓN}

Las expectativas fueron observadas en distintos momentos durante la ejecución. Para ello se utilizaron dos grupos focales, en momentos antes y después, más los testimonios recogidos de los docentes en los círculos de reflexión. En el grupo focal de entrada fue evidente que los docentes tenían un conocimiento parcial y poco claro sobre la investigación-acción, además de una percepción que entendía la problematización como un proceso externo a las prácticas docentes, que eventualmente podría sumarse a lo que el docente hace si hay una justificación para ello. La auto-inspección de la práctica no era percibida como una necesidad, menos como una prioridad, o como un componente esencial en la enseñanza. Sumado a lo anterior -y quizá explicado en parte por ello- se observó poca 
disposición para cambiar las prácticas si esos cambios suponían incorporar elementos de problematización. Esto porque los docentes dudaban de sus capacidades para emplear herramientas de investigación en la enseñanza, o por cierto escepticismo con respecto al aporte que podría significar la investigaciónacción en la mejora de la docencia, o simplemente porque reportaban no contar con el tiempo necesario para ello.

Algunos de los testimonios de los docentes exponen sus razones:

'Yo empleo la problematización. Hablo de mí no de mis colegas. Yo me cuestiono, identifico problemas, trabas, "cuellos de botella" que les dicen en la docencia que hago. Siempre trato de hacer las cosas mejor, busco soluciones y me planteo retos. Claro, lo hago informalmente, fuera del trabajo, cuando estoy en mi casa [...] Creo que tiene alguna utilidad. Claro que si uno no tiene los conocimientos de lo que va a enseñar o no maneja las metodologías necesarias, no va a enseñar bien, por más que sea consciente de los problemas que tiene, de sus limitaciones. Creo que eso es lo principal, luego uno puede plantearse cuestionamientos y retos' (Primer círculo de reflexión: Docente 5).

'Puede ser que reflexionar con los estudiantes y buscar soluciones a los problemas que se pueden presentar en esta relación de nosotros como docentes con ellos, buscar un diálogo, un conocimiento cercano de los problemas que los chicos puedan tener, sea importante; seguro que lo es, pero la prioridad son los contenidos. Si yo tengo que elegir entre uno y otro elijo desarrollar todos mis contenidos y hacerlo bien, porque sé que estos muchachos no van a hacer las dos cosas, muchos docentes tampoco podrían, no se van a hacer bien las cosas, al menos los muchachos no podrían' (Grupo focal de entrada: Docente 12).

Los testimonios parecieran representar la manera como los docentes construyen sus modelos de práctica docente deseable o de sesión de clase, donde la transmisión de contenidos aparece como un fin esencial, que no deja espacios para el cuestionamiento sobre si la manera en que se enseña es la más eficiente o no, diluyendo la posibilidad de evaluar los niveles de efectividad de distintas maneras de enseñar y tomar decisiones a partir de ello. Iniciativas de ese tipo no son percibidas como algo importante o a veces ni siquiera son percibidas. No se tiene conciencia, ni menos ideas claras, del papel que la investigación podría jugar en la identificación de problemas de la docencia y en sus soluciones, o en la contextualización de la enseñanza.

Hacia el final de la ejecución, en el cuarto círculo de reflexión y el grupo focal de salida, la poca expectativa sobre el empleo de la investigación en la docencia se mantuvo. Ningún docente argumentó a favor de la investigación, sea por escepticismo o por desconocimiento. Menos aún se reportó alguna iniciativa o experiencia de reflexión conjunta con los estudiantes, en el sentido de incorporar elementos de indagación en el currículo.

\section{Discusión}

Los resultados sugieren que un programa de investigación-acción con docentes en servicio favorece en el corto plazo el fortalecimiento de habilidades de investigación que tienen en el conocimiento de conceptos y procedimientos una parte importante de su sustento, pero no mejora las expectativas sobre el empleo de la investigación en la docencia. Los resultados también sugieren que la posibilidad de ocurrencia de estos cambios es mayor si en la capacitación se incluye espacios para el cuestionamiento de conocimientos y creencias.

El análisis cuantitativo muestra que entre los momentos antes y después las siete habilidades evaluadas se ven fortalecidas con valores que expresan incrementos significativos en todos los casos. Del mismo modo, la competencia de investigación como totalidad también se fortalece significativamente. Estas mejoras son consistentes con lo hallado en distintos estudios sobre experiencias que emplearon metodologías de investigación-acción para fortalecer capacidades en el manejo de didácticas (Blanchard et al., 2009; Herrington et al., 2011; Megowan-Romanowicz, 
2010; Ruiz-Mallen et al., 2010; Silverstein et al., 2009). Al parecer el empleo de estas metodologías en sí mismo lleva al desa-rrollo de ciertas habilidades asociadas con la investigación como la problematización, el empleo de evidencias, la argumentación, la comunicación, etc., además de favorecer actitudes proclives a la reflexión y la auto-inspección de lo que se hace (Justi \& Van Driel, 2005). Sin embargo, no hay que perder de vista que la evaluación auténtica de cualquier competencia se debe dar en sus ambientes naturales de manifestación, donde cotidianamente se la pone en práctica, y que desde ese punto de vista, esta experiencia no cubrió ese requisito. En este caso, se trata de una evaluación limitada a los conocimientos y a su aplicación en ambientes artificiales, más no de una evaluación de prácticas en aula.

El análisis cualitativo reporta que los docentes dieron muestras de haber asumido una actitud más favorable a la auto-inspección de la práctica. Dejaron de ver a los problemas como situaciones que vienen de afuera de la escuela e influyen en lo que se puede hacer y lograr, para entenderlos como desajustes entre aquello que vienen haciendo y lo que se requiere hacer, dadas las características y necesidades de los grupos a los que se atiende. Con ello, los docentes recuperaron la capacidad de intervenir en la mejora de sus prácticas, puesto que ubicaron los problemas en su zona de influencia, ampliando sus posibilidades de intervención con soluciones que al ejecutarse debían producir simultáneamente mejoras en la enseñanza.

Las intervenciones mismas pasaron a ser entendidas no sólo como soluciones o acciones de mejora de aquello que ocurre en el aula, sino también como procesos de aprendizaje y de desarrollo profesional, mientras que el empleo de la investigación en la docencia en apoyo de esos procesos de fortalecimiento empezó a tener sentido para el docente mismo. Estos resultados son consistentes con la literatura en el campo que informa sobre experiencias que emplearon metodologías de investigación-acción y que estarían explicando cambios importantes en el fortalecimiento de las habilidades de identificación y explo- ración de problemas en el aula (Yamin-Ali, 2010) o en el desarrollo del pensamiento reflexivo del docente con fines de mejora de sus prácticas (Halim, Buang \& Meerah, 2010).

Paralelamente, las propuestas de intervención y las hipótesis que se formularon se fueron alineando con el nuevo tratamiento dado a los problemas. La posición de inicio sobre los problemas y sus soluciones al parecer respondían a las creencias que traían los docentes sobre cómo debe ser un estudiante para tener una experiencia escolar exitosa, o sobre qué tipo de familias son las que tienen mayor probabilidad de asegurar ello, o en qué entornos la educabilidad se facilita (López, 2009). Así las soluciones previstas partían de una visión estereotipada de las situaciones negativas y contenían una alta carga de prejuicios, produciendo interferencia en las valoraciones que los docentes hacen de sus estudiantes, opiniones que enseguida daban sustento a las asignaciones de confianza o a las expectativas de desempeño futuro, incidiendo directamente en las decisiones que finalmente se tomaban. El cambio hacia una actitud más favorable a la problematización de la propia práctica, favoreció el planteo de soluciones dentro del ámbito de la acción docente, dándole intencionalidad pedagógica y viabilidad a las propuestas de intervención. Estos resultados son consistentes con lo reportado en un estudio colaborativo con docentes universitarios y escolares llevado a cabo en Chile (Gonzales-Weil et al., 2013).

En el tema de las expectativas no se registraron cambios importantes. El docente tiene expectativas muy limitadas sobre el papel de la investigación en la docencia, y según los testimonios dados por ellos, dos razones lo explicarían: una primera asociada al desconocimiento sobre el potencial de la investigación para mejorar la docencia, hecho reportado en otros estudios y parte de cuya literatura está consignada a lo largo de este artículo. Una segunda que tiene que ver con las creencias que los docentes traen, que son construcciones esencialmente basadas en la recuperación de las propias vivencias como estudiantes o como docentes, que dan prioridad a una enseñanza discursiva, vertical y autojustificada, 
donde el cuestionamiento de lo que se hace y la participación del estudiante en ello y en las decisiones que se pudieran tomar, no tiene espacio. Como sostienen Herrington y colaboradores (2011), es poco lo que se puede cambiar a nivel de las prácticas, de lo que se hace, si no hay cambios previos a nivel de las creencias, de lo que se piensa. Esto nos lleva a preguntar, ¿En qué medida las creencias docentes manifiestan distintos niveles de resistencia al cambio, ya sea que se trate de cambios en los conocimientos, destrezas o prácticas? ¿En qué medida estas resistencias son variables al margen que los cambios hayan sido impulsados desde espacios abiertos al diálogo y al cuestionamiento? Los cambios han sido significativos a nivel de conocimientos y habilidades, pero no a nivel de las expectativas de empleo de esos conocimientos y habilidades en la mejora continua de la docencia. En ningún testimonio es posible hallar una reflexión sobre los propósitos de la investigación en la enseñanza, situación que hubiese supuesto que el docente cambió en sus expectativas, aclarando el para qué le serviría la investigación y el cómo se la tendría que insertar en la práctica docente. En este tema, los hallazgos del estudio realizado no son consistentes con lo reportado por la investigación previa. Un estudio en profundidad liderado por Blanchard y colaboradores (2009) comunicó cambios en las concepciones sobre la investigación de los docentes de la muestra y en cómo insertar la investigación en el trabajo en aula, mientras un proyecto de investigaciónacción colaborativa a cargo de González-Weil y colaboradores (2013) reportó que los docentes comenzaron a percibir a la investigación como una posibilidad de desarrollo profesional.

Por otro lado, en términos de diseño, el estudio tuvo limitaciones de origen que si bien estuvieron previstas en el inicio, no por ello dejaron de tener importantes consecuencias en sus alcances. Una primera limitación se refiere al hecho de restringir la observación de lo ocurrido a la ejecución del componente de investigación-acción, una intervención corta (10 semanas) que no incluyó el acompañamiento a los docentes en su lugar de trabajo en momentos posteriores, ni la observación de sus prácticas en aula. Todo fue hecho y todo fue observado en los ambientes artificiales de una capacitación fuera de aula, sabiendo que los cambios adquieren sentido cuando se materializan en prácticas, y que éstas sólo pueden ser observadas en sus ambientes naturales de manifestación. En ese marco se tomó la decisión de que el estudio sólo evaluara cambios en conocimientos, habilidades y expectativas, dejando de lado el importante campo de las prácticas. Se sugiere que a futuro la evaluación tome un corte más longitudinal, incorporando momentos adicionales de observación que permitan la evaluación de las prácticas de los docentes en aula.

Una segunda limitación tiene que ver con el hecho de no contar con un grupo de comparación que nos permitiese probar que los cambios observados en habilidades y expectativas se explicaban por el componente de investigación-acción y no por el Programa de Especialización e incluso, por otras intervenciones previas a las que los docentes pudieron haber estado expuestos. Con respecto a esta limitación, se sugiere que en experiencias futuras se trabaje con un grupo control, siempre que sea materialmente posible, porque es evidente que contar con ese referente hubiese supuesto fortalecer grandemente el diseño.

Sin embargo, y pese a las limitaciones expuestas, se considera que los hallazgos dejan algunas recomendaciones que se podrían tomar en cuenta: (1) implementar espacios de reflexión y de cuestionamiento, de diálogo y de logro de acuerdos en los procesos de capacitación, porque son mecanismos probados para superar aquellas creencias sobre la enseñanza que muchas veces llevan a la distorsión de los hechos, (2) implementar sistemas de acompañamiento en aula, incluso en momentos posteriores a la capacitación, en el entendido que la práctica de la enseñanza es el espacio natural para desarrollar habilidades de reflexión sobre la práctica misma y (3) trabajar con el tiempo necesario para procesar y madurar los cambios, principalmente aquellos que tienen que ver con actitudes, expectativas o valores, que por su complejidad toman tiempos más largos en ser internalizados. 


\section{CUADRO 1}

DIMENSIONES, MÓDULOS Y HABILIDADES

\begin{tabular}{|l|l|l|}
\hline \multicolumn{1}{|c|}{ Dimensiones } & \multicolumn{1}{|c|}{ Habilidades } & \multicolumn{1}{c|}{ Módulos } \\
\hline & $\begin{array}{l}\text { Identificación de problemas* } \\
\text { Redacción científica** } \\
\text { Búsqueda de información y evidencias** } \\
\text { Empleo de fuentes, informantes y técnicas* }\end{array}$ & Búsqueda de información y evidencias \\
$\begin{array}{l}\text { Habilidades de } \\
\text { investigación } \\
\text { Análisis de causalidad* } \\
\text { Hipótesis de acción* } \\
\text { Estrategias de intervención* }\end{array}$ & Análisis de causas \\
\hline $\begin{array}{l}\text { Expectativas de } \\
\text { empleo de la } \\
\text { investigación }\end{array}$ & & \\
\hline
\end{tabular}

*Habilidad propia de la metodología de investigación-acción

${ }^{* *}$ Habilidades transversales 


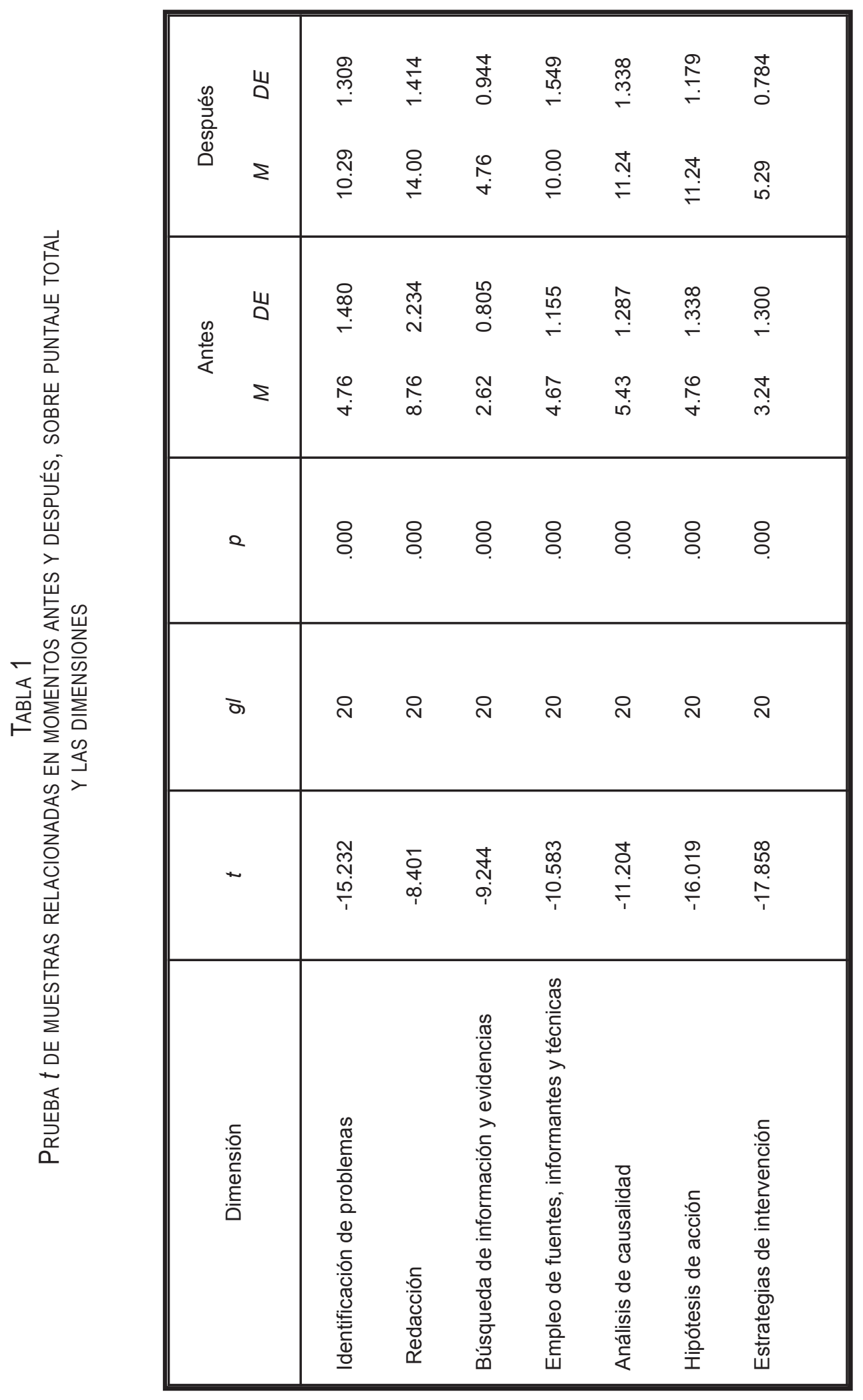




\section{REFERENCIAS BIBLIOGRÁFICAS}

Aranguren, G. (2007). Systemizing action research as an intervention and teacher training strategy in its research role. Revista de Pedagogía, 28 (82), 173-195.

Blanchard, M.R., Southerland, S.A. \& Granger, E.M. (2009). No silver bullet for inquiry: Making sense of teacher change following an inquiry based research experience for teachers. Science Education, 93(2), 322-360. http://dx. doi.org/10.1002/sce.20298

Carr, W. \& Kemmis, S. (1988). Critical theory of teaching. Action research in teacher training. Barcelona: Ediciones Martínez Roca.

Chacón, M.A. Chacón, T. \& Alcedo, Y. (2012). Interdisciplinary learning projects in teacher training. Revista Mexicana de Investigación Educativa, 17(54), 877-902.

Chamizo, J.A. \& García-Franco, A. (2013). Heuristics diagrams as a tool to formatively assess teachers' research. Teachers and teaching: Theory and practice, 19(2), 135-149. http://dx.doi. org/10.1080/13540602.2013.741841

Díaz, C., Villalón, M. \& Adlerstein, C. (2015). Conocimiento práctico para la enseñanza del lenguaje oral y escrito de estudiantes de educación inicial: Una aproximación desde la elaboración de mapas conceptuales [Prospective early childhood teachers' practical knowledge to teach oral language and literacy: An approach from conceptual mapping]. Interdisciplinaria, 32(2), 331-345. https://doi.org/10.16888/interd.2015. 32.2 .8

Fernandes, C.H. (2014). Relationships between research and permanent teacher training: The necessary knowledge for diversity. Estudios Pedagógicos, 40(2), 161-174. http://dx.doi.org/ $10.4067 / \mathrm{S} 0718-07052014000300010$

García Fraile, J. \& Tobón Tobón, S. (2008). Management of the curriculum by competences. An approach from the complex systemic model. Lima: AB Representaciones.

González-Weil, C., Cortez, M., Pérez, J.L., Bravo, P. \& Ibaceta, Y. (2013). Building encounter domains to problematize secondary science teacher's practices: Incorporating the action-research model as a continuous training plan. Estudios Pedagógicos, 39(2), 129-146. http:// dx.doi.org/10.4067/S0718-070520130002000 09

Graebner, I.T., de Souza, E.M.T.D. \& Saito, C.H. (2009). Action-research and food and nutrition security: A school experience mediated by conceptual graphic representation tool. International Journal of Science Education, 31(6), 809-827. http://dx.doi.org/10.1080/09500690 701885646

Halim, L., Buang, N.A. \& Meerah, T.S. (2010). Action research as instructional supervision: Impact on the professional development of university based supervisors and science student teachers. Procedia-Social and Behavioral Sciences, 2(2), 2868-2871. https://doi.org 10.101 6/j.sbspro.2010.03.430

Hernández, R., Fernández-Collado, C. \& Baptista, P. (2007). Metodología de la investigación [Research methodology]. México: McGraw-Hill / Interamericana.

Herrington, D.G., Yezierski, E.J., Luxford, K.M. \& Luxford, C.J. (2011). Target inquiry: Changing chemistry high school teachers' classroom practices and knowledge and beliefs about inquiry instruction. Chemistry Educational Research and Practice, 12(1), 74-84. http://dx.doi.org/1 0.1039/C1RP90010B

Ibernón, F. (2001). Training in schools: Trend or fashion? En F. López (Comp.), Teacher training: Training projects in schools (pp. 15-22). Barcelona: Grao.

Justi, R. \& Van Driel, J. (2005). A case study of the development of a beginning chemistry teacher's 
knowledge about models and modelling. Research in Science Education, 35(2-3), 197-219. http://dx.doi.org/10.1007/s11165-004-7583-z

Laudadío, J. (2012). Evaluación de estilos de enseñanza en la universidad: Estudio preliminar de las propiedades psicométricas del Cuestionario sobre la Orientación Docente del Profesor Universitario (CODPU) [Evaluation of teaching styles at college: Preliminary study of the psychometric properties of the Questionnaire on the Teaching Orientation of the University Professor]. Interdisciplinaria, 29(1), 79-93. https:// doi.org/10.16888/in terd.2012.2 9.1.5

López, N. (Coord.). (2009). Relationships, actors and territories. Towards new education policies in Latin America. Buenos Aires: International Institute for Education Planning, Unesco.

Maarof, N. (2007). Telling his or her story through reflective journals. International Education Journal, 8(1), 205-220. Recuperado el 25 de febrero de 2016 de http://ehlt.flinders.edu. au/education/iej/articles/v8n1/Maarof/paper.p df

Mckernan, J. (1996). Action research and curriculum. Madrid: Morata.

Megowan-Romanowicz, C. (2010). Inside out: Action research from the teacher-researcher perspective. Journal of Science Teacher Education, 21(8), 993-1011. http://dx.doi.org/10.100 7/s10972-010-9214-z

Miles, M. \& Huberman, A.M. (1984). Qualitative data analysis. A sourcebook of new methods. Beverly Hills: Sage Publications.

Mitchener, C.P. \& Jackson, W.M. (2012). Learning from action research about science teacher preparation. Journal of Science Teacher Education, 23(1), 45-64. http://dx.doi.org/10.1007 /s 10972-011-9261-0

Montecinos, C., Solis, M. \& Gabriele, A. (2001). Learning to teach through action research. Paideia Revista de Educación, 30(31), 37-50.
Park Rogers, M., Abell, S., Lannin, J., Wang, Ch., Musikul, K., Barker, D. \& Dingman, S. (2007). Effective professional development in science and mathematics education: teachers' and facilitators' views. International Journal of Science and Mathematics Education, 5(3), 507-532. http://dx.doi.org/10.1007/s10763-006-9053-8

Perrenoud, P. (2004). Developing reflective practice. Barcelona: Grao.

Rodríguez-Sosa, J. (2005). Education action research. What is it about? How is it carried out? Lima: Doxa.

Rodríguez, N. (2004). Challenges of teacher training in Venezuela. Revista de Pedagogía, 25(73), 3-12.

Ruiz-Mallen, I., Barraza, L., Bodenhorn, B., CejaAdame, M.D.L.P. \& Reyes-García, V. (2010). Contextualising learning through the participatory construction of an environmental education programme. International Journal of Science Education, 32(13), 1755-1770. http://dx. doi.org/101080/ 09500690903203135

Silverstein, S.C., Dubner, J., Miller, J., Glied, S. \& Loike, J.D. (2009). Teachers' participation in research programs improves their students' achievement in science. Science, 326(5951), 440-442. http://dx.doi.org/10.1126/science.117 7344

Slafer, G. (2009). ¿Cómo escribir un artículo científico? [How to write a scientific article?]. Revista de Investigación en Educación, 6, 124132.

Tobón, S. (2005) Competences in higher education: Policies towards quality. Bogotá: ECOE.

Vaillant, D. (2009). Secondary school teachers training: Realities and speeches. Revista de Educación, 350, 105-122. Recuperado el 18 de marzo de 2016 de http://www.revistaeducacio n.mec.es/re350/re350_05.pdf

Yamin-Ali, J. (2010). Translating concerns into action in the foreign language classroom. $G E$ 
MA Online Journal of Language Studies, 11(2), 21-38. Recuperado el 23 de mayo de 2016 de http://ejournal.ukm.my/g ema/article/view/54
Zeichner, K. (2001). Educational action research. En P. Reason \& H. Bradbury (Eds.). Handbook of action research. Participative inquiry and practice (pp. 273-283). London: Sage.

Facultad de Educación Universidad Peruana Cayetano Heredia (UPCH) Lima-Perú

Fecha de recepción: 21 de junio de 2016 Fecha de aceptación: 17 de enero de 2017 